\title{
RECENT STUDIES OF THE BRIGHTEST STARS \\ IN THE MAGELLANIC CLOUDS
}

\author{
PATRICK S. OSMER \\ Cerro Tololo Inter-American Observatory* , La Serena, Chile
}

\begin{abstract}
The objective prism surveys carried out by Sanduleak $(1968,1969)$ make available for the first time a relatively complete and homogeneous list of the brightest stars in both Magellanic Clouds for the range of spectral types O-G5. Photometric observations in the four color, $\mathrm{H} \beta$ system of the 169 candidates in the SMC and the 45 brightest stars in the LMC as well as spectroscopic observations of the most luminous early-type stars, lead to the following picture of the stability and evolution of very massive stars:

(1) The reddening is the same for the most luminous stars in both Clouds and there is a sharply defined upper limit to the stellar luminosity, which is also the same in both Clouds. This limit corresponds to $M_{\text {bol }}=-11 \pm 1$ or $90 \mathfrak{M}_{\odot}$, in good agreement with the classic study of Feast et al. (1960).

(2) The stars of highest total luminosity are the blue supergiants, and in both Clouds the high temperature limit corresponds to type $\mathrm{B} 1.5$ or $T_{\mathrm{e}} \sim 25000 \mathrm{~K}$ for $M_{v} \sim-8$. The SMC appears to be deficient in luminous F5-G5 supergiants.

(3) With few exceptions the most luminous stars are not known to show more than low amplitude variations in light. They do show $\mathrm{H} \beta$ emission as expected, and four stars in the LMC have anomalously strong emission, indicative of unusual atmospheric activity. Bright Wolf-Rayet stars have long been known in the Clouds; in addition, several candidate Of stars are indicated by the photometry.

(4) The brightest B-type supergiants in the SMC appear to be deficient in N, Si, O, and probably $\mathrm{Mg}$ and $\mathrm{C}$, although helium is normal, a result worth pursuing in the less luminous stars.

(5) Judging from the numbers of the brightest stars, the recent rate of star formation in both galaxies per unit mass is quite similar despite the fact that the SMC has a larger fraction of neutral hydrogen and the LMC a more conspicuous display of HII regions.
\end{abstract}

\section{References}

Feast, M. W., Thackeray, A. D., and Wesselink, A. J.: 1960, Monthly Notices Roy. Astron. Soc. 121, 337. Sanduleak, N.: 1968, Astron. J. 73, 246.

Sanduleak, N. : 1969, Cerro Tololo Inter-American Obs. Contr. No. 89.

* Operated by the Association of Universities for Research in Astronomy. Inc., under contract with the National Science Foundation. 


\section{DISCUSSION}

Bessell: In view of your comment that these stars are likely to be helium-burning stars, I would like to draw your attention to John Robertson's models of a B0Ia supergiant of $60 M_{\odot}$, still in the hydrogenburning phase. For older clusters in the Magellanic Clouds with turn off masses of $15 M_{\odot}$, the existence of blue supergiants is probably due to the blue straggler process.

Osmer: I think you have to consider the time scale problem too, though. It must put some kind of restriction on how long they can burn hydrogen. I suppose this question will be answered by the number of main sequence stars we do find.

Hyland: Can you explain the observed slope of the upper limit of observed luminosities of Magellanic Cloud stars in the $M_{\mathrm{BOL}}-\log T_{\mathrm{e}}$ diagram?

Osmer: I think it's real, in that we surely know whether there are stars in this region of the diagram. However, the bolometric corrections are imprecise.

Irwin: Is there any information as to binaries or percentage of binaries in those stars near the upper limit of luminosity in your diagram. It would be nice to know the percentage of binaries and, of course, individual masses of some of these stars.

Osmer: There is very little evidence that I know of. I agree, it would be nice to know what fraction of the stars are binaries.

Irwin: I'd like to make just a comment. Many years ago, Shapley identified many of the brightest red stars in both clouds; he called these stars superluminous stars.

Przybylski: You said that stars in the Magellanic Clouds appear to have normal helium lines. There is one danger that one defines the spectral type of hot stars according to the strength of helium lines and in fact it may well be that there may be some stars which are actually hotter but helium weak. We cannot exclude this possibility.

Osmer: That was a good question. I just happened to be ready for it. I looked at that quite carefully in these B stars. I was concerned about whether this was all consistent or not. Silv lines are quite strong in B0 and then drop right off quite quickly; Si III lines come up at B1, and then you get Sill coming up at B2. With the ratio of the silicon lines you can really pin down the temperature because things happen quickly here and then on the same scale, the other lines - let's say helium or carbon or nitrogen - most of them vary rather slowly. The final bit of information is of course the colour. I found that in fact you could get a consistent colour and spectral type from the silicon lines and then examine whether the helium and carbon and nitrogen and so on, were normal. Everything did work out consistently and the helium was identical to the stars in the Large Cloud and the one in Scorpius as far as I can tell. I agree about your point, but in these particular stars I think there is some reason to believe the helium is normal.

Parsons: Have you looked at the $m_{1}$ index, and if so, do you notice any systematic difference between the SMC and LMC?

Osmer: I have looked at the $m_{1}$ index, and so far, I have not notice any systematic differences but I've not pursued it too much. It's a difficult question for these very luminous stars simply because of a lack of good calibration. I think there's some useful data there; I haven't gone into it too far other than to note that it appears to be similar but I think you'd have to work at it fairly hard to get a meaningful result out of that. 